\title{
Ganaderización novohispana en el norte del Obispado de Michoacán, siglos XVI-XVII
}

\author{
New-hispanic cattle-ranching in the north of the Bishopric of \\ Michoacan, 16th-17th centuries
}

\author{
América A. Navarro-López ${ }^{1}$ \\ Pedro S. Urquijo-Torres ${ }^{2}$ \\ Gerardo A. Hernández-Cendejas ${ }^{3}$ \\ Universidad Nacional Autónoma de México, México
}

\section{Resumen}

En este trabajo analizamos los procesos históricos y geográficos de la ganadería en el norte del obispado de Michoacán a través de un análisis cartográfico, en el periodo que va de 1550, una vez aplicadas las Nuevas Leyes, y hasta 1650, fecha posterior a la puesta en marcha de las Composiciones de tierra (1643). Metodológicamente recurrimos al análisis de documentos de la época, tales como licencias de mercedes para estancias de ganado (mayor y menor) así como a los informes obispales, con la finalidad de procesarlos y elaborar cartografía a partir de Sistemas de Información Geográfica orientados a la Historia (SIG-Histórico). Lo anterior nos permite espacializar los lugares en que se establecieron las estancias de ganado. Si bien la atribución de otorgar licencias de ganado correspondía a la autoridad civil, el obispado veía trastocado sus intereses territoriales en jurisdicciones bajo su responsabilidad eclesiástica. Investigaciones como la presente abordadas desde la historia

1 Dra. Universidad Nacional Autónoma de México. Centro de Investigaciones en Geografía Ambiental. Antigua carretera a Pátzcuaro No. 8701, ExHacienda de San José de la Huerta, C.P. 58190, Morelia, Michoacán, México.anavarro@pmip.unam.mx

2 Dr. Universidad Nacional Autónoma de México. Centro de Investigaciones en Geografía Ambiental. Antigua carretera a Pátzcuaro No. 8701, ExHacienda de San José de la Huerta, C.P. 58190, Morelia, Michoacán, México.psurquijo@ciga.unam.mx

3 Dr. Universidad Nacional Autónoma de México. Escuela Nacional de Estudios Superiores- Morelia. Antigua carretera a Pátzcuaro No. 8701, ExHacienda de San José de la Huerta, C.P. 58190, Morelia, Michoacán, México.ghercendejas@enesmorelia.unam.mx

Este artículo corresponde a la ponencia presentada en el 35th Conference of Latin American Geographers realizada en San José, Costa Rica del 20 al 22 de mayo del 2018. 
y la cartografía histórica, son cada vez más pertinentes, ya que cubren vacíos importantes en la historiografía de la región de estudio, misma que ha dejado al margen la perspectiva geográfica.

Palabras clave: ganadería, obispado de Michoacán, SIG-H, estancias, diezmo.

\begin{abstract}
In this work we analyze the historical and geographical processes of animal husbandry in the north of the Bishopric of Michoacán through a cartographic analysis of the period from 1550, when the New Laws were applied, to 1650, just after the implementation of the Composición de Tierras (1643). Methodologically, we undertook analysis of historical documents, such as land grants for livestock raising (large- and small-scale) and bishopric reports, to process them and elaborate their cartography in a Geographic Information System oriented to historical analysis (Historical GIS). This allows us to spatially situate the establishment of cattle ranches. Although the granting of cattle licenses rested in the purview of the civil authority, the bishopric viewed this as a disruption of the territorial interests in jurisdictions under its ecclesiastical responsibility. Research such as this, that deals with history and historical cartography, are becoming increasingly relevant, as they address important lacunae in a historiography of the study region that has largely relegated the geographical perspective to the margins.
\end{abstract}

Keywords: Bishopric of Michoacan, animal husbandry, (ecclesiastic) land grants, tithe, H-GIS

\title{
Introducción
}

El proceso de ganaderización novohispano del siglo XVI se caracterizó, principalmente, por ser de carácter extensivo y, por tanto, determinante en la transformación del paisaje. Sobre esto último, su estudio ha sido abordado en diferentes investigaciones con distintos énfasis; por ejemplo, el cambio ambiental, la tenencia de la tierra, la adaptación y manejo de paisaje y los cambios en los usos de suelo (Melville, 1994; Aguilar-Robledo, 2001; Sluyter, 2001, Hunter, 2013).

El hecho de que el otorgamiento de mercedes, a partir de las Nuevas Leyes de $1542^{4}$, proporcionara derechos de propiedad sobre la tierra, hizo que fueran un incentivo que animó a los colonizadores a incursionar en territorios norteños considerados por los europeos como agrestes, debido a la constante incursión de indios belicosos denominados genéricamente como chichimecas. El argumento para la ocupación de ese espacio fue percibirlo como vacio, a pesar de la existencia de los grupos nativos, que, al no contar con elementos tangibles de propiedad de la tierra, quedaban eliminados a la vista de los agentes irruptores (Sheridan, 2015).

4 Las Nuevas Leyes de Indias, aluden a una revisión exhaustiva del régimen de encomiendas, con dos finalidades, la primera de ellas fue dar protección a los indios, mediante la supresión de la esclavitud, pero también buscaban disminuir el poderío político y económico que los encomenderos habían logrado construir en el territorio novohispano.

384 Revista Geográfica de América Central № 61E (3) Especial CLAG Este artículo está bajo una licencia e-ISSN 2215-2563 • Julio-diciembre 2018 • pp.383-395 Atribución/Reconocimiento-NoComercialDoi: http://dx.doi.org/10.15359/rgac.61-3.19

CompartirIgual 4.0 Internacional 
En el caso particular del septentrión del Obispado de Michoacán, la ganadería fue de tipo extensiva; es decir, aquella que se practicaba en amplios espacios y que atendía grandes cantidades de ganado. Las estancias de ganado mayor eran para ganado vacuno; sin embargo, en la práctica, era común que estos espacios se utilizaran para caballos, yeguas y mulas. Mientras que las estancias de ganado menor se referían a ganado ovino, y en ellas, además de ovejas, solían verse también cabras y cerdos.

El establecimiento de las estancias sirvió de apoyo tanto para el crecimiento jurisdiccional del obispado, como para el abastecimiento de los centros mineros en la ruta de la plata. También fortalecía el abasto en la ruta hacia las minas del septentrión del obispado michoacano, específicamente las ubicadas en la Villa de Guadalcazar. Los estancieros se convirtieron en agentes importantes para la consolidación y expansión del territorio, de la mano de la evangelización y la apertura de caminos, ya que donde se abría un camino, se fijaban estancias y, en muchos de los casos, las estancias servían también como misión (Elías, 2014).

En este artículo representamos cartográficamente, a partir de Sistemas de Información Geográfica orientados a la Historia (SIG-Histórico), las mercedes de tierra asociadas a la ganadería en el norte del obispado de Michoacán, mediante el análisis de documentos de la época. Para el siglo XVI se recurrió a los Manuscritos 1121 de la Biblioteca Newberry de Chicago en su Colección Ayer, y, Manuscrito 140 de la Biblioteca del Congreso de Washington, en su colección Kraus, compilados por Paredes (1994). Para el siglo XVII se trabajó con la Relación de la Diócesis de Michoacán del Obispo Fray Baltasar de Covarrubias del año 1619, procedente de la Biblioteca del Antiguo Palacio Real de Madrid, en la sección "Manuscritos de América", y compilada por Lemoine (1993); la Minuta de las Doctrinas y Beneficios del Obispado de Michoacán, de 1631, autoría que se atribuye a Fray Francisco de Rivera, que fue localizada en la Secretaría del arzobispado de la catedral de Morelia y compilada por el clérigo López ( 1973); la Demarcación y descripción del Obispado de Michoacán, de 1648, hecha por el canónigo Fray Arnaldo de Y sassy y compilada en el volumen I, número 1 de la Biblioteca Americana (1982).

Consideramos que es importante estudiar la región en cuestión, ya que puede observarse una relación entre la expansión de su jurisdicción, vía el otorgamiento de mercedes para estancias, y la administración del 
diezmo durante el periodo de estudio. Además, con este tipo de abordajes, se contribuye en el esclarecimiento de un vacío importante en la historiografía de la región, misma que ha dejado al margen la perspectiva geográfica o espacial.

El trabajo se compone de tres apartados. En el primero, se presentan las características geográficas del septentrión del obispado de Michoacán en el periodo de estudio. En el segundo apartado, explicamos la metodología utilizada para la elaboración del SIG-Histórico. Finalmente, damos cuenta de los resultados obtenidos.

\section{Área de estudio}

El septentrión del obispado de Michoacán se localizaba en una región de límites no muy bien definidos, mismos que fueron ampliándose en el transcurso de la segunda mitad del siglo XVI y la primera del XVII. Las primeras estancias ganaderas en la región de estudio se pueden ubicar en el Bajío, una extensa llanura aluvial que forma el río Lerma y sus afluentes, concentrándose en el corredor que iba de la Villa de San Miguel a Comanja (Lagos de Moreno). Siguiendo hacia el septentrión se encuentran las primeras estribaciones de la Sierra Madre Oriental y es allí donde ubicamos la parte más septentrional del obispado, en la Villa de Guadalcazar y la custodia del Río Verde. (Mapa 1)

Conforme fue avanzando la Conquista también lo hizo la construcción de la jurisdicción episcopal del obispado michoacano. Es así como, para mediados del siglo XVI, se establecen los límites septentrionales en la Villa de San Miguel por el oriente, y la Villa de León por el rumbo occidental. (Mapa 1) 
América A. Navarro-López, Pedro S. Urquijo-Torres, Gerardo A. Hernández-Cendejas. Ganaderización novohispana en el norte del Obispado de Michoacán, siglos XVI-XVII

Mapa 1. El obispado de Michoacán en la Nueva España, siglo XVI

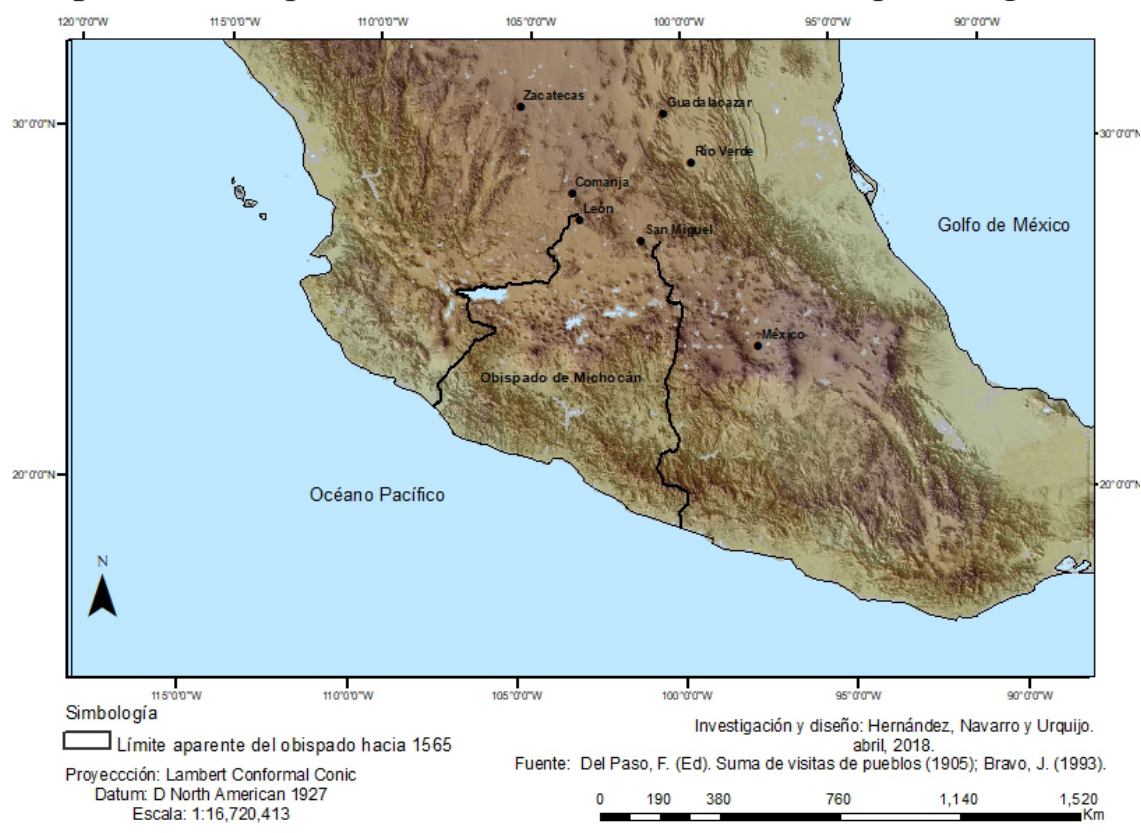

Dado que el espacio es dinámico y cambia constantemente, esos aparentes "límites" se fueron recorriendo hacia el norte, y la estrategia de otorgamiento de mercedes, para estancias de ganado fue muy acertada en ese sentido.

Para 1619, de acuerdo con el informe del obispo Fray Baltasar de Covarrubias, en la Villa de San Felipe hay "muchas estancias" (Baltasar en Lemoine, 1993:181), descripción que da cuenta del avance jurisdiccional, que oscila [casi a la mitad] entre el paralelo 21 y 22.

En 1630, en la Minuta que se atribuye al obispo Rivera (López, 1973) se mencionan estancias, ranchos y haciendas que practicaban actividades ganaderas en Los Pozos, Valle de San Francisco, Pueblo y Frontera de Santa María de Atotonilco y San Miguel Mezquitic. Pasando, con poco, el paralelo 22. Para 1648, el límite máximo lo ubicamos en Real de Minas de Armadillo.

\section{Procesamiento metodológico}

A partir del análisis de documentos de la época sobre licencias y mercedes de tierras entre 1551 y 1553 , y los informes de los obispos en 1619, 1631 y 1648, elaboramos un SIG histórico. Identificamos espacialmente 
los lugares en que estaban establecidas las estancias de ganado mayor y menor. Lo anterior nos llevó a observar patrones espaciales de cercanía entre ellas, de cercanía también a los ríos, y de cercanía también, a caminos importantes. Para proceder a la realización de la cartografía, analizamos las fuentes documentales históricas, localizando los datos que nos son de utilidad para la temática abordada en nuestra región de estudio. Construimos la base de datos (geodatabase) que procesamos en Arc GIS.

Lo siguiente fue pasar a la etapa de análisis de la dinámica espacial, con diferentes capas (shapefile) de información del Instituto Nacional de Estadística Geografía e Informática (INEGI), con la finalidad de ubicar los lugares y asignarles un punto en nuestro primer mapa. Un problema al que nos enfrentamos al trabajar fuentes del siglo XVI y XVII con una base de datos cartográfica del siglo XXI, fue el de localizar de manera certera la ubicación de los lugares. En ese sentido, tuvimos que corroborar si un pueblo cambió o no de lugar, o bien si cambió su toponimia. Cuando tuvimos dudas al respecto, la forma en que procedimos fue regresar a la narración de la fuente primaria, poniendo especial atención en la descripción del medio físico, para poder contrastarlo con la imagen de un mapa actual, y de esta manera fijar el punto en donde se encontraba ubicado.

Lo descrito anteriormente nos lleva a elaborar el primer mapa de puntos, mismo que nos sirve para contrastar las preguntas de investigación que tenemos, pero no sólo eso, sino que, al considerar al mapa como una herramienta analítica, surgen nuevas preguntas de investigación y se exploran nuevas hipótesis. Proyectamos este mapa en un MDE a 90m, en el shape de cuerpos de agua de INEGI, y uno de caminos elaborado con base en Bakewell (1976).

El resultado es una cartografía analítica de la que surgen nuevas preguntas de investigación y qué, nos ayuda a observar patrones espaciales para los diferentes momentos que se otorgaron mercedes, de acuerdo con las fuentes históricas, para las estancias de ganado mayor y menor en el septentrión del obispado michoacano.

\section{Resultados}

Analizar espacialmente la dinámica de las estancias ganaderas en el norte del obispado michoacano nos brindó la posibilidad de explorar nuevas hipótesis y observar patrones que, debido a la ausencia de este tipo de estudios en la historiografía michoacana, quizás habían pasado desapercibidos. 
América A. Navarro-López, Pedro S. Urquijo-Torres, Gerardo A. Hernández-Cendejas. Ganaderización novohispana en el norte del Obispado de Michoacán, siglos XVI-XVII

Los patrones son de cercanía entre las estancias, además de cercanía a ríos y caminos, estos últimos, no sólo en dirección a la Ruta de la Plata o de Tierra Adentro, la cual ha sido abordada con demasía en la historiografía colonial minera, sino que detectamos la existencia de caminos secundarios que conectaban a otros centros mineros que quedaban dentro de la jurisdicción del obispado de Michoacán y que se ubicaban en su límite más septentrional. Específicamente nos referimos a la Villa de Guadalcazar y su zona aledaña. (Mapa 2)

Mapa 2. Estancias ganaderas y rutas mineras en el obispado de Michoacán, siglos XVI-XVII

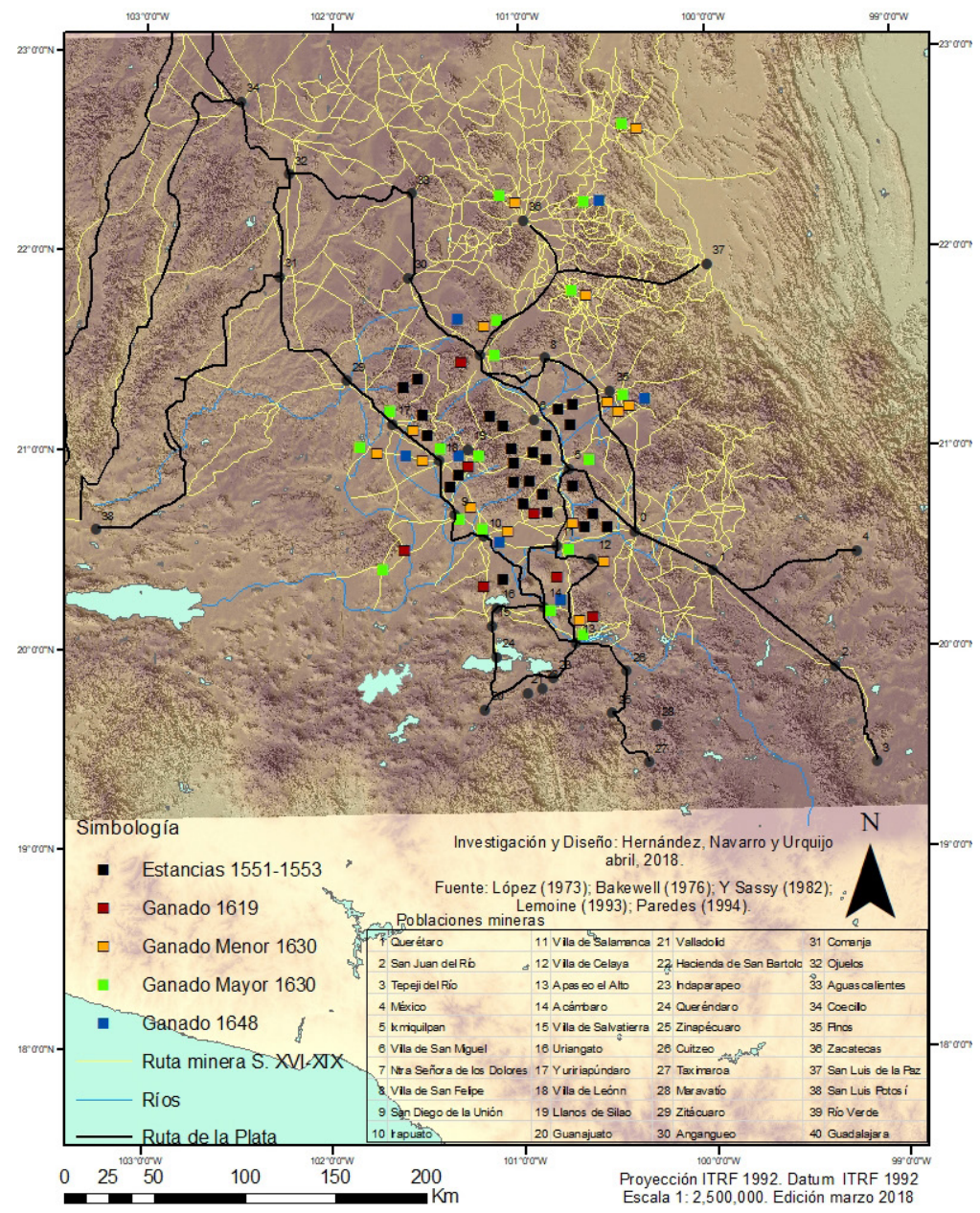


Identificamos, como a partir del otorgamiento de mercedes desde el siglo XVI, se fue creando una red de intereses políticos y económicos que beneficiaba. Por ejemplo, a familiares de virreyes, generales de guerra -o en caso de muerte a sus familiares-, primeros colonizadores, religiosos, y, a funcionarios administrativos. Vemos el nacimiento de una cúpula de grupos de poder integrada por los nuevos pobladores del norte michoacano, incentivados, seguramente por la riqueza que la actividad ganadera generó durante las primeras décadas en territorio novohispano.

Por lo que toca a la administración del diezmo, notamos como todavía hasta fines del siglo XVI y las primeras tres décadas del XVII los estancieros del septentrión del obispado michoacano, no estaban obligados a diezmar, esto como estrategia de estímulo desde la autoridad virreinal para la ocupación de ese complejo territorio. Es a partir de 1630 que en los informes obispales se menciona que lugares como Yuririapúndaro, o al Valle de San Francisco diezmaban con ganado (López, 1973). (Mapa 2). Por lo que toca a los diezmos por producción agrícola es hasta 1636 que se tiene registro de estancias en León e Irapuato que diezmaron por la cantidad de 1918.88 el primero y 48 pesos el segundo (Florescano, 1987). (Mapa 3). Esto, mientras se lograba la pacificación del territorio chichimeca, vía la evangelización, reducción y conversión de Pames, Guamares y Guachichiles. 
América A. Navarro-López, Pedro S. Urquijo-Torres, Gerardo A. Hernández-Cendejas. Ganaderización novohispana en el norte del Obispado de Michoacán, siglos XVI-XVII

Mapa 3. Estancias ganaderas y diezmo en el obispado de Michoacán, 1630-1648

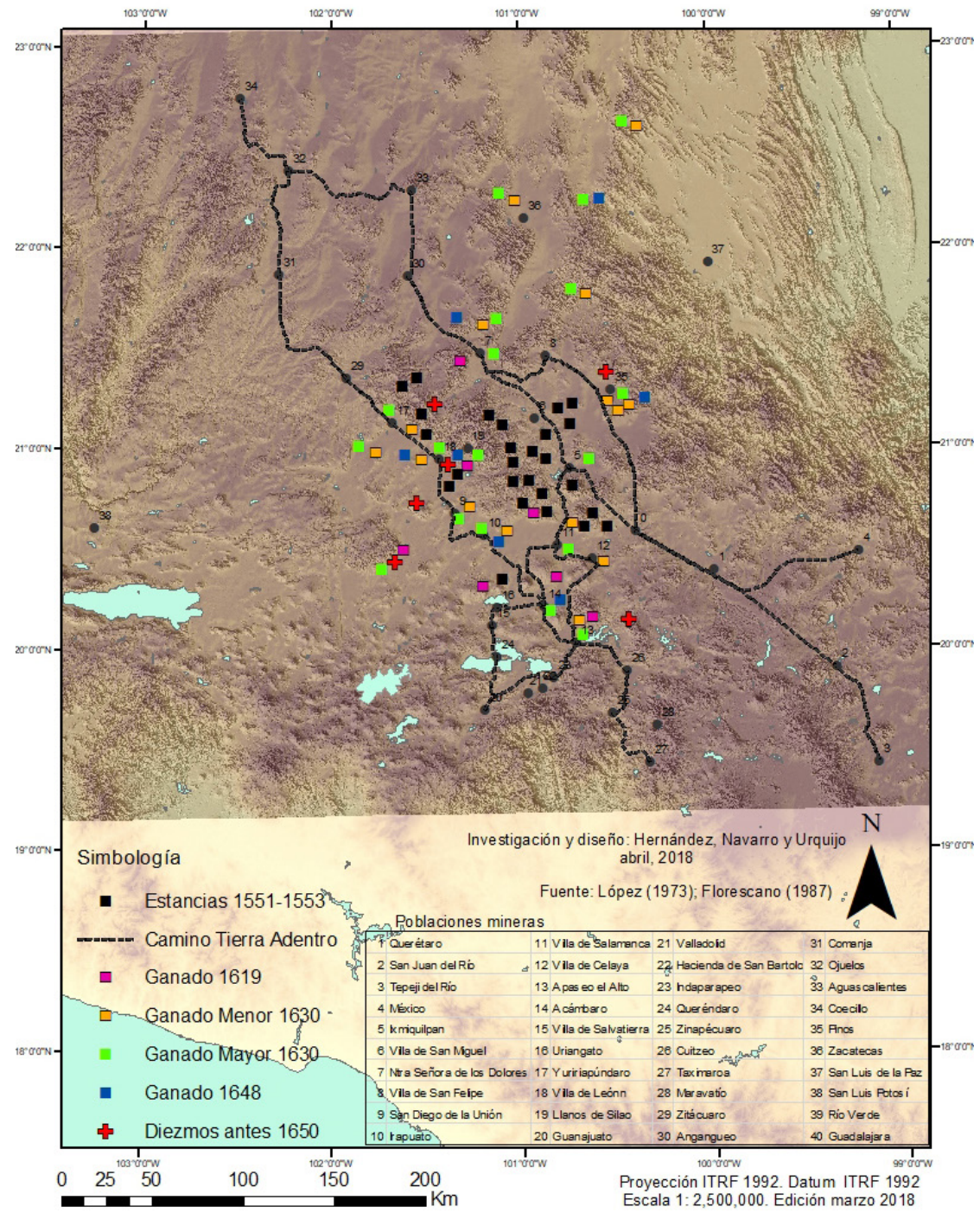

La información que nos proporcionó el primer mapa de puntos, nos permite plantearnos nuevas preguntas de investigación con hipótesis que apuntan a rumbos distintos a los explorados hasta ahora.

Vemos como el Septentrión del obispado se expande entre el siglo XVI y XVII novohispanos, sostenido en una estrategia, caracterizada por 
el proceso de ganaderización vía otorgamiento de mercedes. En un primer momento, observamos el crecimiento jurisdiccional por la parte oriente, que va de Acámbaro- Yuririapúndaro, hacia Celaya, Apaseo y Chamacuero, San Miguel, Los Pozos y Santa María de Atotonilco. Por el occidente el avance ganadero fue de Pénjamo hacia El Rincón, la Villa de León, San Felipe, más hacia el norte llegó hasta el Valle de San Francisco y Mezquitic (mapa 3), este último lugar, con sólo dos estancias propiedad de dos mujeres, Doña María de Gordezuela y Doña María de Medina, seguramente viudas de generales de guerra, en donde la fuente nos reporta, de veinte a treinta mil ovejas para el primer caso y "grosedad de ganado" para el segundo (López, 1973). Imaginemos ese paisaje, que, a decir de la fuente, estaba habitado sólo por "doscientos y cincuenta indios", conviviendo y compartiendo el espacio con la cantidad de ganado que mencionamos.

Habría que señalar que el establecimiento de estancias ganaderas, y por tanto, la ampliación jurisdiccional del obispado no sucedió de manera uniforme o lineal, fue más complicado y lento avanzar por la parte occidental, dada la presencia de los grupos Guamares y Guachichiles.

Las características del terreno y el desconocimiento del mismo fueron factores que complicaban la ocupación ibérica. Los conquistadores avanzaban enfrentando a grupos altamente entrenados para la guerra, sofisticados en el uso del arco y la flecha, y que con el tiempo aprendieron, a utilizar las técnicas y armas traídas de Europa para defender su territorio. Además, los nativos, cazadores y recolectores por excelencia, eran conocedores del terreno en que se movían, situación que les daba cierta ventaja.

El otorgamiento de mercedes para estancias continuaba y los colonizadores avanzaban, de la mano de estrategias evangelizadoras y de guerra. Poco se acordaban ya de fijar nuevos límites. El canónigo Y Sassy, para 1648 nos da indicios al respecto cuando señala que después de las mojoneras puestas en la Villa de León y San Miguel, "no sé han medido en lo que nuevamente se ha ido ganando y descubriendo hacia el norte" (Y Sassy [1648] 1982: 2). De acuerdo con Aguilar (2009), durante la Colonia, el desarrollo de las herramientas y técnicas de medición fue deficiente, quizá esta afirmación pueda contribuir a entender por qué no se iba midiendo lo recién ganado. Sin embargo, para nosotros es más probable que no se haya medido por lo difícil que resultaba ese espacio, en el sentido de que los grupos nativos volvían a establecerse en territorios que se consideraban 
América A. Navarro-López, Pedro S. Urquijo-Torres, Gerardo A. Hernández-Cendejas. Ganaderización novohispana en el norte del Obispado de Michoacán, siglos XVI-XVII

ya "pacificados" y porque el avance no se daba en línea recta homogénea hacia el norte.

Para 1648 se mencionan estancias ganaderas en Salvatierra, Valle de Santiago, Salamanca, Palmar de Vega, todos estos por el centro-oriente. Por el poniente se conserva San Francisco del Rincón, detectamos estancias, ranchos y haciendas de ganado mayor y menor en Marfil, Silao y Armadillo. El Valle de San Francisco se conserva como centro estanciero, reportando estancias con una actividad que oscilaba, ya desde 1630, entre 20 mil y 100 mil cabezas de ganado, y una población de apenas "seis cientas personas grandes y pequeñas” (López [1630] 1973:57). (Mapa 3)

Algo sobresaliente es que las fuentes del siglo XVII, no sólo mencionan estancias, sino también haciendas y ranchos que practicaban la actividad ganadera. Lo que nos remite a Chevalier (1976:137) en el sentido de que fueron las estancias la etapa previa a la formación de la hacienda. Para nosotros va más allá de ver a la estancia por sí misma como punto de partida o explicativo. Más bien ésta, formó parte de la estrategia virreinal de apropiación territorial y evangelización, a partir del otorgamiento de mercedes, en nuestro caso de estudio para estancias de ganado mayor y menor, y esta estrategia, fue el punto de inicio para la formación de las grandes propiedades de hacendados y rancheros novohispanos, que se complementó perfectamente con las composiciones de tierra de 1643, que van a definir el rumbo de la propiedad rural en el resto del periodo novohispano, y, sin lugar a dudas ayudarán a determinar los tipos de propiedad agraria que permanecerán a lo largo del tiempo.

Haber abordado el tema de la ganaderización novohispana desde una perspectiva de análisis espacial nos permitió observar eventos que desde la historiografía tradicional no han sido vistos, precisamente porque se ha dejado de lado el enfoque espacial. Nos permite también, abonar a un relato con nuevas variables, que cuenta con un rigor metodológico aceptable, a partir de las herramientas que nos aportan los Sistemas de Información Geográfica.

\section{Referencias}

Acuña, R. (1987). Relaciones Geográficas del siglo XVI: Michoacán. México: Instituto de Investigaciones Antropológicas-UNAM.

Aguilar-Robledo, M. (2001). "Ganadería, tenencia de la tierra e impacto ambiental en la Huasteca Potosina: los años de la Colonia”. Historia 
ambiental de la ganadería en México. Xalapa, México: Instituto de Ecología, AC. (pp.9-24)

Aguilar-Robledo, M. (2009). "Contestred terrain: the rise and decline of surveying in New Spain, 1500-1800". En Journal Latin American Geography, 8(2), 23-47.

Bakewell, P. (1976) Minería y sociedad en el México colonial. Zacatecas (1546-1700). México: Fondo de Cultura Económica.

Chevalier, F. (1976). La formación de los latifundios en México. México: Fondo de Cultura Económica.

Elías, F. (2014). La colonización ganadera en el Valle de los Chichimecas. La comarca y el desarrollo de una élite ganadera, 1540-1610. Guanajuato, México: Forum Cultural Guanajuato.

Guzmán, C. (2017). "Investigación histórica, los SIG y las nuevas posibilidades epistemológicas y metodológicas". En Urquijo, P., Vieyra, A., Bocco, G. (2017). Geografía e Historia Ambiental. Morelia, México: Centro de Investigaciones en Geografía Ambiental-UNAM.

Hernández, L. (Compiladora) (2001). Historia ambiental de la ganadería en México. Xalapa, México: Instituto de Ecología.

Hunter, R. (2014). Land Use Change in New Spain: A Three-Dimensional Historical GIS Analysis. En: The Professional Geographer, 66(2), 260-273.

Instituto Nacional de Estadística Geografía e Informática. (1992). Topónimos por localidades.

Lemoine, E. (1993). Relación de la Diócesis de Michoacán hecha por el Obispo Fray Baltasar de Covarrubias en Valladolid en 1619, Versión Paleográfica. En Valladolid-Morelia 450 años. Documentos para su historia 1537-1828. Morelia, México: Morevallado Editores.

López, R. (ed.) (1973). Minuta y Rrazon de las doctrinas qye ay en este Obispado de Mechuacan assi beneficios de clerigos como gudarnias y prioratos de rreligiosos de San Francisco y San Agustin con los pueblos y feligreses que cada doctrina tiene. Versión Paleográfica. En El obispado de Michoacán en el siglo XVII. Informe inédito de beneficios, pueblos y lenguas. Morelia, México: FIMAX publicistas. Melville, E. (1994). Plaga de ovejas: consecuencias ambientales en la conquista de México. México: Fondo de Cultura Económica. 
América A. Navarro-López, Pedro S. Urquijo-Torres, Gerardo A. Hernández-Cendejas. Ganaderización novohispana en el norte del Obispado de Michoacán, siglos XVI-XVII

Paredes, C. (ed.) (1994). Y por mi visto...mandamientos, ordenanzas, licencias y otras disposiciones virreinales sobre Michoacán en el siglo XVI. México: Universidad Michoacana de San Nicolás de Hidalgo/Centro de Investigaciones y Estudios Superiores en Antropología Social/Secretaría de Educación Pública.

Sheridan, C. (2015). Fronterización del espacio hacia el norte de la Nueva España. México: CIESAS/ Instituto Mora.

Sluyter, A. (1998). The ecological origns and consequences of cattle ranching in sixteenth-century New Spain. En: Geographical Review, 86(2), 161-77.

Sluyter, A. (2001). "Ganadería española y cambio ambiental en las tierras bajas de Veracruz, México, siglo XVI". Historia ambiental de la ganadería en México. Xalapa, México: Instituto de Ecología, AC. (pp.25-40)

Y Sassy, A. (1982). "Demarcación y Descripción de El Obispado de Mechoacan y Fundación de su Iglesia Catedral. En Bibliotheca Americana, I(1). Florida, University of Miami Station, Coral Gables. 
